\title{
Total cross section of furfural by electron impact: Experiment and theory
}

A. Traoré Dubuis, A. Verkhovtsev, L. Ellis-Gibbings, K. Krupa, F. Blanco, D. B. Jones, M. J. Brunger, and G. García

Citation: The Journal of Chemical Physics 147, 054301 (2017);

View online: https://doi.org/10.1063/1.4996462

View Table of Contents: http://aip.scitation.org/toc/jcp/147/5

Published by the American Institute of Physics

\section{Articles you may be interested in}

Excited state wavepacket dynamics in $\mathrm{NO}_{2}$ probed by strong-field ionization

The Journal of Chemical Physics 147, 054305 (2017); 10.1063/1.4996461

Total cross sections for electron scattering by 1-propanol at impact energies in the range 40-500 eV

The Journal of Chemical Physics 147, 194307 (2017); 10.1063/1.5008621

Next generation extended Lagrangian first principles molecular dynamics

The Journal of Chemical Physics 147, 054103 (2017); 10.1063/1.4985893

Low-energy electron-induced dissociation in gas-phase nicotine, pyridine, and methyl-pyrrolidine

The Journal of Chemical Physics 147, 094303 (2017); 10.1063/1.4994679

Quaternionic formulation of the two-component Kohn-Sham equations and efficient exploitation of point group symmetry

The Journal of Chemical Physics 147, 054101 (2017); 10.1063/1.4995614

Dissociation dynamics of transient anion formed via electron attachment to sulfur dioxide

The Journal of Chemical Physics 147, 054304 (2017); 10.1063/1.4994899

\section{A $\mid$ The Journal of Chemical Physics}




\title{
Total cross section of furfural by electron impact: Experiment and theory
}

\author{
A. Traoré Dubuis, ${ }^{1, a)}$ A. Verkhovtsev, ${ }^{1}$ L. Ellis-Gibbings, ${ }^{1}$ K. Krupa, ${ }^{1}$ F. Blanco, ${ }^{2}$ D. B. Jones, ${ }^{3}$ \\ M. J. Brunger, ${ }^{3}$ and G. García ${ }^{1, b)}$ \\ ${ }^{1}$ Instituto de Física Fundamental, Consejo Superior de Investigaciones Científicas, Serrano 113-bis, \\ 28006 Madrid, Spain \\ ${ }^{2}$ Departamento de Física Atómica, Molecular y Nuclear, Universidad Complutense de Madrid, \\ 28040 Madrid, Spain \\ ${ }^{3}$ School of Chemical and Physical Sciences, Flinders University, GPO Box 2100, Adelaide, \\ South Australia 5001, Australia
}

(Received 24 May 2017; accepted 17 July 2017; published online 1 August 2017)

\begin{abstract}
We present experimental total cross sections for electron scattering from furfural in the energy range from 10 to $1000 \mathrm{eV}$, as measured using a double electrostatic analyzer gas cell electron transmission experiment. These results are compared to theoretical data for furfural, as well as to experimental and theoretical values for the structurally similar molecules furan and tetrahydrofuran. The measured total cross section is in agreement with the theoretical results obtained by means of the independentatom model with screening corrected additivity rule including interference method. In the region of higher electron energies, from $500 \mathrm{eV}$ to $10 \mathrm{keV}$, the total electron scattering cross section is also estimated using a semi-empirical model based on the number of electrons and dipole polarizabilities of the molecular targets. Together with the recently measured differential and integral cross sections, and the furfural energy-loss spectra, the present total cross section data nearly complete the data set that is required for numerical simulation of low-energy electron processes in furfural, covering the range of projectile energies from a few electron volts up to $10 \mathrm{keV}$. Published by AIP Publishing. [http://dx.doi.org/10.1063/1.4996462]
\end{abstract}

\section{INTRODUCTION}

Furfural is an important molecule in green chemistry and in the agricultural, petrochemical, and processing industries. ${ }^{1}$ This key chemical is derived from lignocellulose species, ${ }^{2}$ and its applications include oil refining, a substitute for petrochemicals, pharmaceuticals, and agrochemical industrial work. Furfural is used directly in furan resins and in the extraction of unsaturated compounds and impurities from alkanes. ${ }^{3}$ Formed in the thermochemical treatment of biomass, production estimates were as large as $2.1 \times 10^{5}$ tons per year ${ }^{3}$ in 2013 , mainly synthesized via catalysis from xylan derived from bagasse and corn cob.

Atmospheric pressure plasma or electron beam irradiation processes are used as pre-treatments of biomass in order to generate a higher yield conversion to biofuels. ${ }^{4,5}$ This, amongst other modeling plasma applications, ${ }^{6}$ suggests a need for accurate and comprehensive electron-furfural collision models. ${ }^{7} \mathrm{~A}$ further rationale for the present work is to provide measured data in order to benchmark and expand reaction databases for ionized gases such as recently proposed by Tennyson et al. ${ }^{8}$

A heterocyclic aldehyde, furfural $\left(\mathrm{C}_{5} \mathrm{H}_{4} \mathrm{O}_{2}\right)$, is closely related to furan and tetrahydrofuran (THF), their 5-member rings constituting some of the smallest aromatic species (see Fig. 1). The cis and trans conformations of the aldehyde branch exist in a $20.5 \%-79.5 \%$ ratio in the gas phase, though it has

\footnotetext{
a) Electronic mail: traoredubuis@iff.csic.es

b) Electronic mail: g.garcia@csic.es
}

been shown theoretically that the differences in the elastic cross sections between the conformations is minimal. ${ }^{9}$ Furfural, furan and THF can also be considered as analogues ${ }^{10}$ for the sugar backbone of the DNA helix-deoxyribose. It is now well known that radiobiological damage comes in great part from reactions with the low-energy secondary electrons and radicals resulting from the primary ionizing radiation. ${ }^{11-13}$

With both industrial and medical applications, low-energy electron-furfural interactions and particle track modeling are therefore of high importance. ${ }^{14}$ Monte Carlo particle track simulation codes that work specifically in the low-energy region [such as the Low Energy Particle Tracking Simulation (LEPTS) code developed by our research group ${ }^{15,16}$ ] require both interaction cross sections and experimental energy-loss profiles for incident electron energies below $10 \mathrm{keV}$. It is well known that in electron-molecule collisions with an energy lower than $10 \mathrm{keV},{ }^{16}$ the first Born approximation no longer holds and so will not reliably estimate the electron scattering cross sections of the molecules in question. For this reason, some experiments based on crossed beam ${ }^{17}$ gas cell ${ }^{18}$ and magnetically confined ${ }^{19}$ electron beam techniques have been employed to determine experimental differential and integral elastic and inelastic cross section data. These data are then used to test the validity of model approaches such as the Independent-Atom Model with Screening Corrected Additivity Rule including Interference (IAM-SCAR+I), ${ }^{20}$ as well as ab initio calculations employing close-coupling ${ }^{21}$ and R-matrix ${ }^{22}$ methods. Together, experimental and theoretical contributions are able to help us to form complete 

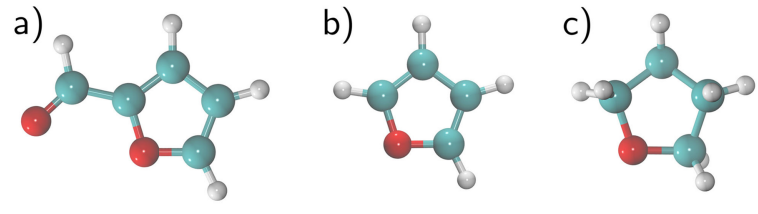

$\mathrm{C}_{5} \mathrm{H}_{4} \mathrm{O}_{2}$

$\mathrm{C}_{4} \mathrm{H}_{4} \mathrm{O}$

$\mathrm{C}_{4} \mathrm{H}_{8} \mathrm{O}$

FIG. 1. Representation of the structure of the furfural (a), furan (b), and THF (c) molecules which we have considered in this study.

or near-complete electron scattering cross section data sets for molecules of interest to varied sections of the scientific community.

To try to fully understand, and subsequently model, the electron-furfural interactions, the low-energy electronmolecule collision community has been collaborating internationally to produce a set of experimental and theoretical reference data. For example, experimental studies of the electronic $^{7,23}$ and vibrational ${ }^{9}$ excitation differential and integral cross sections for $20-40 \mathrm{eV}$ incident electrons were compared to theoretical results obtained by means of the Schwinger multichannel method with pseudopotentials (SMCPP), IAMSCAR $+\mathrm{I}$, and binary-encounter-Bethe (BEB) methods. In addition, the experimental triple differential cross sections (TDCS) for ionization with $250 \mathrm{eV}$ electron impact and a $21.22 \mathrm{eV} \mathrm{He}$ (I) photoelectron spectrum, along with calculations within the molecular 3-body distorted wave framework ${ }^{24}$ have also been reported. Electron energy-loss (EEL) and vacuum ultraviolet (VUV) spectroscopy ${ }^{25}$ studies have also been undertaken in the $3.5-10.8 \mathrm{eV}$ photon energy region and at 20 , 30 , and $250 \mathrm{eV}$ electron energies and compared to the minimal orbital basis single configuration interaction (MOB-SCI) ab initio method results to elucidate the states observed. Where possible, these experiments were also compared to theoretical calculations for furfural or experiments for furan. Note that in modeling studies, it is vital to include as much of the scattering phenomena as possible and, in particular, to separate discrete inelastic excitations from each other and from the ionization channel. ${ }^{26}$ A recent summary of the available furfural cross section can be found in Ref. 27.

The total cross section (TCS), being the sum of the elastic and inelastic scattering cross sections, for all open channels, of the molecular target, as a function of energy, ultimately serves as the upper boundary for theoretical models and as a check for the consistency of the partial integral cross sections. In particular, for theories that provide elastic and inelastic integral cross sections (ICSs), the sum of those cross sections of all channels should match the TCS to within the experimental errors. ${ }^{28}$ As far as we know, no extensive experimental total cross sections for furfural are available with only limited results (at 7, 10, and $20 \mathrm{eV}$ incident energies) from Lozano et al. ${ }^{29}$ having been previously reported. However, the SMCPP method was used to calculate the elastic ICSs, the momentum transfer cross sections, and the TCS in the energy range $5-50 \mathrm{eV}^{30}$

Comparisons for furan and THF are relevant and have been reported in the literature to investigate the role of molecular structure in scattering phenomena; here we do the same. The first reported measurements of the electron scattering TCS from THF molecules were from Mozejko et al. ${ }^{31}$ using the linear electron-transmission method in the $1-370 \mathrm{eV}$ range. The TCS for furan was also reported by the same group, ${ }^{32}$ although now for electron incident energies from 0.6 to 400 $\mathrm{eV}$. The TCS from THF has also been measured quite recently by other groups: Zecca et al. ${ }^{33}$ reported TCS data for the electron energy range of $2-21 \mathrm{eV}$, while Baek et al. ${ }^{34}$ measured the TCS and elastic differential cross sections (DCSs) for electrons in the energy range from $20 \mathrm{eV}$ to $1 \mathrm{keV}$. Finally, measurements of the TCS from THF molecules were performed by Fuss et al. ${ }^{35}$ and covered a broad electron energy range of 50-5000 eV.

In this paper, we report experimental measurements of the TCS for electron scattering from furfural in the impact energy range from 10 to $1000 \mathrm{eV}$. The experiments were carried out at the Instituto de Física Fundamental, CSIC, Madrid, using a double electrostatic analyzer gas cell electron transmission experiment. We compare results from these measurements to theoretical data for furfural and further with results for the furan and THF molecules. Together with the recently measured differential and integral cross section and the energy loss spectra (Refs. 7, 9, 23, 25, 29, and 30), the present TCS data provide a nearly complete dataset for numerical simulation of low-energy electron processes in furfural.

The remainder of this paper is organized as follows. In Sec. II, we present the experimental configuration and measurement details, including a discussion on our uncertainties. Section III briefly details both the IAM-SCAR+I method ${ }^{20}$ and the semi-empirical model, ${ }^{36}$ whose results are used to compare with our measured data. Our results and a discussion of these results are presented in Sec. IV, followed by some conclusions drawn from this work.

\section{EXPERIMENTAL METHODS}

\section{A. Experimental details}

The total scattering cross section, $\sigma_{\text {tot }}(E)$, describes the probability of an incident electron, of specified kinetic energy $E$, interacting with a target molecular system over a solid angle and encompasses all open scattering processes, namely, elastic and inelastic events. For a transmission experiment, $\sigma_{\text {tot }}$ can be deduced from the Beer-Lambert law

$$
I=I_{0} \exp \left(-n L \sigma_{\mathrm{tot}}\right),
$$

where $I_{0}$ is the intensity of the primary electron beam, $I$ is the transmitted intensity, $n$ is the number density of the biomolecular gas, and $L$ is the collision length which to first order is taken as the geometrical length of the collision chamber. In our experiments, $L$ is equal to $5 \mathrm{~cm}$. The molecular density $n$ of the furfural gas is retrieved from the measured pressure $P$, using an MKS Baratron absolute capacitance manometer, as follows from the ideal gas law: $n=P / k_{\mathrm{B}} T$, where $k_{\mathrm{B}}$ is the Boltzmann constant and $T$ is the gas temperature which is assumed to be equal to the room temperature $(300 \mathrm{~K})$.

A recently installed double electrostatic analyzer experimental system, for measuring electron scattering dynamics, performs the TCS measurements. We note that a similar apparatus was used by Hannay et $a l^{37}$ and Duflot et al. ${ }^{38,39}$ for valence and inner shell electronic spectroscopy of molecules 


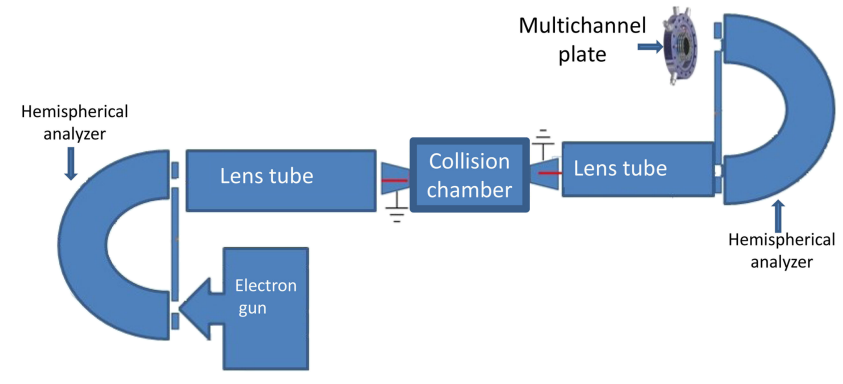

FIG. 2. Schematic diagram of the double electrostatic analyzer used to perform the total cross section measurement for furfural.

in the gas phase. A schematic diagram of the experimental configuration is shown in Fig. 2.

The apparatus consists of five regions: (i) an electron gun, (ii) an initial hemispherical analyzer, (iii) the collision chamber, (iv) a second hemispherical analyzer, and, finally, (v) the electron detection region. All sections of the equipment have mu-metal (a nickel-iron magnetic alloy with very high permeability) shielding in order to reduce the effects of the external magnetic fields. Electrons generated by thermionic emission from a filament are directed towards the hemispherical analyzer in order to produce a monochromatic beam of electrons with an energy spread around $0.5 \mathrm{eV}$ (full width half maximum). The theoretical relationship between the electron's energy traversing the monochromator and the electrostatic potential applied across the hemispherical deflectors is given by 40

$$
E=\Delta V /\left(\frac{R_{\mathrm{o}}}{R_{\mathrm{i}}}-\frac{R_{\mathrm{i}}}{R_{\mathrm{o}}}\right),
$$

where $R_{i}$ and $R_{o}$ are the radii of the inner and outer hemispheres, respectively, and $\Delta V$ is the difference in voltage applied to the inner and outer hemispheres. Using the values corresponding to our experiment, $R_{i}=72 \mathrm{~mm}$ and $R_{o}=128$ $\mathrm{mm}$ in Eq. (2), one selects the final kinetic energy in the units of $\mathrm{eV}$ for electrons reaching the gas sample by $E=1.21 \Delta V$. The angular acceptance is determined solely by the selected magnification mode, which is defined by physical apertures in the lens tube attached to the analyzer. In our apparatus, a four-element electron lens attached to both analyzers defines the angular acceptance of $1.15^{\circ}$ over the whole energy range. ${ }^{41}$ This scattering angle is kept relatively constant due to the presence of mu-metal covering the vacuum vessel as well as the electron spectrometers. Deflection plates for $\mathrm{x}$ and $\mathrm{y}$ electron beam steering are located in both lens tubes, and they direct the beam to the entrance of the collision chamber and second hemisphere, respectively. Note that the monochromator and collision chamber are differentially pumped.

The collision chamber is a simple gas cell $(50 \times 50 \times 50$ $\mathrm{mm}^{3}$ ), with entrance and exit holes of $1 \mathrm{~mm}$ diameter. To achieve the kinetic energy desired, the gas cell is either biased with an external power supply to accelerate or decelerate the approaching electrons or kept electronically grounded. The gas sample is introduced directly into the collision chamber using an effusive technique. After passing through the gas cell, electrons traveling within the 0.02 rad angle enter a hemispherical analyzer which in principle serves to discriminate the scattered electrons based on their energy. This is a crucial functionality in terms of measuring the EEL spectra. Finally, the transmitted electrons are detected by a 2-stage Hamamatsu micro-channel plate (MCP) operating in single pulse mode. The electron kinetic energy hitting the target is first determined by the power supply connected to the filament. Then, if necessary, the collision energy is further varied using an additional power supply connected to the gas cell by a feedthrough. With this technique, electrons are further accelerated when using a positive power supply (HCN 14-12 500 FuG Elektronik $\mathrm{GmbH}$ ) or retarded when using a negative power supply (FC5P24-220 Glasmann High Voltage). Note that increasing or decreasing the electron energy using this approach may also distort the electromagnetic field inducing a deviation of the primary beam. As a consequence, the total cross section measurements have been performed with the incident energy and the scattering angle kept constant, while the transmitted intensity is recorded as a function of pressure in the gas cell during the collision processes.

A further experimental precaution was that the background vacuum pressure is always kept below $10^{-7}$ Torr to avoid filament contamination during measurements. The analyzer was remotely controlled by a PC running a custom LabView (National Instruments) program in order to record the EEL spectra. The MCP detects single electrons, and the signal is amplified and discriminated to reduce electronic noise. The kinetic energy of the electron beam has been calibrated by measuring the transmitted intensities for different accelerating voltages ranging from 100 to $500 \mathrm{eV}$ with a step of 50 $\mathrm{eV}$. To further evaluate error sources, in particular, how well the scattering length in Eq. (1) is represented by the geometrical length of the collision chamber, we have measured the TCS of a well-studied atom, argon, and compared this with accurate data available in the review paper by Gargioni and Grosswendt. ${ }^{42}$ Note that Gargioni and Grosswendt ${ }^{42}$ did not provide error estimates on their recommended TCS values. Considering the spread of data from different sources included in their compilation, we estimated an uncertainty limit of $\pm 8 \%$. This comparison is given in Table I. As can be seen in this table, the agreement between the two sets of data is within 11\%-16\%. It is also clear from Table I that to within their combined uncertainties they clearly overlap. This gives us some confidence in the validity of our measurement techniques and therefore in their later application to furfural. Nonetheless, it is further clear from Table I that our present argon TCSs are systematically lower than those of Gargioni and Grosswendt. ${ }^{42}$ This observed

TABLE I. Total scattering cross sections of argon (in $\AA^{2}$ ) as measured in this work at several electron energies and used to check the accuracy of our experimental setup. Error bars correspond to one standard deviation. The present results are compared to the digitized reference data from the work of Gargioni and Grosswendt. ${ }^{42}$

\begin{tabular}{llr}
\hline \hline & \multicolumn{2}{c}{$\sigma_{\text {tot }}\left(\AA^{2}\right)$} \\
\cline { 2 - 3 }$E(\mathrm{eV})$ & This work & Reference 42 \\
\hline 133.5 & $6.36 \pm 0.74$ & $7.53 \pm 0.60$ \\
174.5 & $5.79 \pm 0.52$ & $6.65 \pm 0.53$ \\
244.0 & $5.03 \pm 0.39$ & $5.63 \pm 0.45$ \\
\hline \hline
\end{tabular}


difference may reflect that the actual scattering chamber length may be a little different to the geometrical length of the scattering chamber $(L=5 \mathrm{~cm})$, which represents a systematic error in our results that needs to be accounted for. The energy resolution was determined by looking at the nearest distinguishable features of the EEL spectra from molecular nitrogen gas, with the typical resolution being found to be $\sim 0.5 \mathrm{eV}$ (FWHM).

\section{B. Sample}

The furfural liquid sample used in the present experiments was purchased from Sigma-Aldrich with a stated minimum purity of $98 \%$. Dissolved gases were removed from the sample by a repeated freeze-pump-thaw cycle using liquid nitrogen. It was seen that furfural undergoes structural changes, exhibited by a colour change, when exposed to light or air. Therefore, prior to each TCS measurement, the quality of the sample was checked by recording EEL spectra and performing an analysis to ensure the absence of contaminants and confirm the sample stability. Figure 3 shows a typical EEL spectrum of furfural obtained at the collision energy of $433 \mathrm{eV}$ and a scattered electron angle close to $0^{\circ}$. As can be seen in this figure, the energy resolution used for the TCS measurements is good enough to discriminate the attenuation measurements against inelastic channel contaminations but the EEL spectra also provide an additional tool to sample the purity of the target. In addition, recorded EEL spectra produce useful data for the charged particle track simulations.

\section{Measurement protocol}

The furfural pressure in the collision chamber was varied between 1 and 5 mTorr for the TCS measurements, once the background pressure is subtracted. Uncertainty in the absolute pressure value was lower than $1 \%$ according to the instrument data sheet. Measurements were carried out with electron currents ranging from approximately $10^{-11}$ to $10^{-9} \mathrm{~A}$, with typical count rates for TCS measurements being between $10^{2}$ and $10^{4} \mathrm{~s}^{-1}$.

The key parameter for determining the TCS is the recorded transmitted peak intensity. This is measured while keeping the

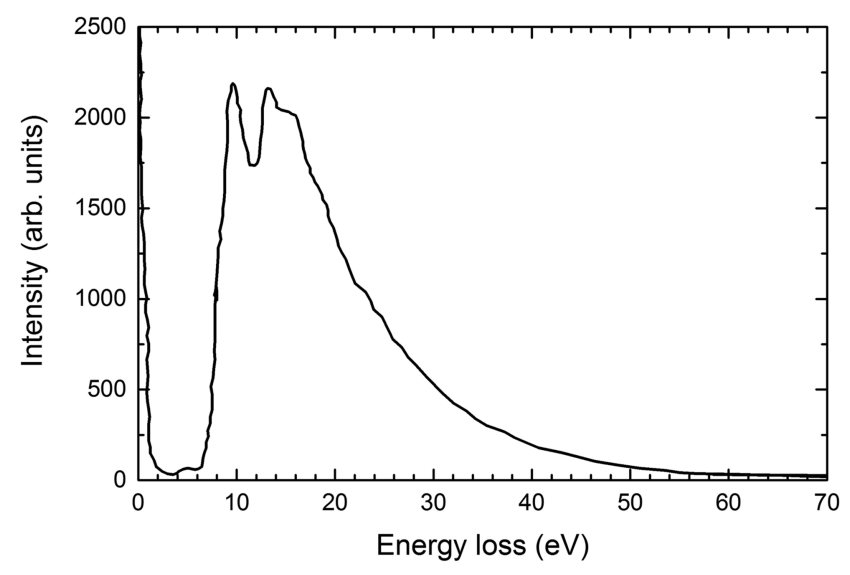

FIG. 3. Typical measured electron energy-loss spectrum from furfural molecules for $433 \mathrm{eV}$ incident energy electrons and close to $0^{\circ}$ electron scattering angle. gas pressure relatively low in order to maintain the singlecollision approximation and therefore the validity of Eq. (1). The procedure is the following. First, we measured the intensity of the primary beam in the absence of the gas target (i.e. $I_{0}$ ). Then, we put between 0.5 and 1 mTorr of the gas of interest into the collision chamber and measured the transmitted intensity. Subsequently, the pressure was increased another 0.5 to 1 mTorr for a third measurement. This step was further repeated until the pressure reached 5 mTorr. At least 10 values of pressure were measured for each cross section, at a given kinetic energy. In addition, each cross section measurement was independently repeated at least three times, so assuring statistical uncertainties below $10 \%$.

\section{Experimental uncertainties}

As noted above, the uncertainty in the pressure value is assumed to be lower than $1 \%$, referring to the manufacturer data sheet (MKS Baratron). The collision chamber is held at room temperature, with its temperature being measured with a thermocouple with an uncertainty of the order of $1 \%$. The incident beam energy was set by a Bertan power supply that exhibits a maximum standard deviation of $1 \mathrm{eV}$ $( \pm 0.5 \mathrm{~V})$ as measured by a Keithley $6517 \mathrm{~A}$ electrometer. This translates to an uncertainty of about $10 \%$ in the energy of the reported TCS value at $10 \mathrm{eV}$ electron kinetic energy and less than $0.2 \%$ above $500 \mathrm{eV}$. Note that in principle this represents an $x$-axis uncertainty bar in the TCS versus energy plots we show later, although in practice this uncertainty limit is not actually plotted. The experimental (statistical) reproducibility in the results of our measurements lies between $0.4 \%$ and $9.3 \%$.

Another source of systematic error in such an apparatus comes from the electrons elastically scattered in the forward direction. In fact, Eq. (1) supposes an infinitely narrow electron beam and a zero detector solid angle. Earlier studies using Monte Carlo simulations showed that the elastic forward scattering contribution in the TCS increases exponentially; however, it was found to be well below $1 \%$ at $3 \mathrm{keV}$ electron energy. ${ }^{36}$ A reasonable maximum error contribution of $0.5 \%$ has therefore been assigned to the elastic contribution in this work. Note that inelastic channels are removed by the hemispherical energy analyzer placed between the scattering chamber and its detection region. We found that one of the main uncertainty sources originates from the sample, which is difficult to handle due to its low vapor pressure, relatively high viscosity $\left(1.49 \mathrm{mPa} \mathrm{s}^{-1}\right)$, and light sensitivity. Furfural is in fact difficult to work with as it is a "sticky" liquid, affecting pressure homogeneity inside the collision chamber. Finally, the aforementioned uncertainty due to our use of the geometric cell length to represent the value of $L$ in Eq. (1) must be considered $(<3 \%)$.

By combining the systematic and random errors, we obtained the total uncertainty limits associated with the present measurements for electron energies between $\sim 10$ and $1000 \mathrm{eV}$. These limits range from $4 \%$ to $22 \%$ depending on the incident energy, with a maximum value of $22 \%$ found for $967 \mathrm{eV}$ impact energy. This percentage encompasses all other sources of uncertainty such as temperature variation, the uncertainty in the true value of $L$ employed in Eq. (1), signal fluctuations due 
to the electronic circuitry, an uncertainty in the curve fitting process, and the filament current instability as well as due to the "stickiness" of liquid furfural.

\section{THEORY}

\section{A. The IAM-SCAR+I method}

To better evaluate our experimental TCS results, we compare these to our previously published results for electron scattering from furfural, ${ }^{7}$ as obtained by means of the Independent-Atom Model with Screening Corrected Additivity Rule and Interference effects (IAM-SCAR+I). The IAMSCAR+I method for the calculation of electron and positron scattering cross sections has been improved recently, as outlined in Ref. 20. This method builds on our previous work, ${ }^{43-45}$ for both electrons and positrons, and has been used successfully in the past for biologically and industrially relevant molecules such as water, ${ }^{46}$ pyrimidine, ${ }^{46} \mathrm{THF}^{35}$ and many more in the range of $1 \mathrm{eV}-10 \mathrm{keV}$ incident energy.

The principle of the IAM-SCAR+I approach is to consider the cross section, $\sigma_{\mathrm{mol}}$, of a molecule as the coherent sum of the screening corrected (reduced) cross sections of the atomic components, with an extra term for the interference contribution to the differential cross section. ${ }^{20}$ We use the optical potential method with each atom in the molecule described by a local complex potential given by

$$
V(r)=V_{\mathrm{s}}(r)+V_{\mathrm{ex}}(r)+V_{\mathrm{pol}}(r)+i V_{\mathrm{abs}}(r),
$$

where the real part comprises the following three terms. $V_{\mathrm{s}}$ is the static term derived from a Hartree-Fock calculation ${ }^{47}$ of the atomic charge distribution, $V_{\mathrm{ex}}$ is an exchange term which accounts for the indistinguishability of the incident and target electrons; it is given by the semi-classical energydependent formula derived by Riley and Truhlar, ${ }^{48}$ and $V_{\text {pol }}$ is a polarization potential for the long-range interactions which depend on the target dipole polarizability, in the form given by Zhang et $a l .{ }^{49}$ Finally, the absorption potential accounts for the electronically inelastic scattering events. It is based on the quasi-free model by Staszewska et al. ${ }^{50}$ but incorporates some improvements to the original formulation, such as the inclusion of screening effects, local velocity corrections, and the description of the electron indistinguishability, ${ }^{51}$ leading therefore to a model which provides a realistic approximation for electron-atom scattering over a broad energy range. ${ }^{52}$

We assume that the independent-atom model additivity rule holds and that the molecular cross section can be built as the sum of the atomic cross sections. As such, we use the common expression for multicenter dispersion

$$
F(\theta)=\sum_{\text {atoms }} f_{i}(\theta) e^{i \mathbf{q} \cdot \mathbf{r}_{i}} .
$$

Here, $\mathbf{q}=\mathbf{k}_{\text {out }}-\mathbf{k}_{\text {in }}$ is the momentum transfer, $\mathbf{r}_{i}$ are the atomic positions, and $f_{i}(\theta)$ are the atomic scattering amplitudes. Subsequent application of the optical theorem results in the "additivity rule" and an inclusion of interference effects that are present in the differential cross section. As the energy of the incoming particle decreases, the additivity rule tends to overestimate the molecular cross section $\sigma_{\text {mol }}$. The factor $s_{i}$ in Eq. (5) is a screening correction, reducing the contribution of each atom to the total molecular cross section $\left(0 \leq s_{i} \leq 1\right)$, which is based on the position of the atom within the molecule and the known total cross section of each atom in the molecule. $\sigma^{\text {int }}$ is the integral of the interference term present in the DCS and provides a higher order approximation of the ICS,

$$
\sigma_{\mathrm{mol}}^{\mathrm{tot}}=\sum_{\text {atoms }} s_{i} \sigma_{\text {atom, } i}^{\mathrm{tot}}+\sigma^{\mathrm{int}} .
$$

This interference term has been found to adjust the values of the TCS significantly across the entire energy range. ${ }^{20,53}$ It arises from the proper treatment of the DCS according to the multi-center scattering equations, whereby a non-vanishing interference term is integrated and remains in the ICS. This appears to point towards a discrepancy in the first-order use of the optical theorem for the ICS.

The key feature behind the IAM-SCAR+I method is that with only the atomic potentials and the atomic spatial coordinates, one can predict the molecular cross section with reasonable accuracy from approximately $1 \mathrm{eV}$ up to $10 \mathrm{keV}$. We have applied this method, as described in our most recent papers, to furfural $^{7}$ and we include it here in order to compare with the new experimental data.

\section{B. A two-parameter semi-empirical model}

The TCSs of furfural, furan, and THF molecules have also been calculated in this work using a semi-empirical model proposed by García and Manero, ${ }^{36,54}$ although these calculations have only been performed for electron impact energies above $500 \mathrm{eV}$.

The semi-empirical model is a relatively quick and computationally cheap method for predicting the TCS of complex molecules. Further it might serve to audit both the theoretical approaches commonly used to estimate the TCS and also the experimental data, in the range above several hundreds of eV. In Refs. 36 and 54, the following two-parameter analytical equation was proposed:

$$
\sigma_{\text {tot }}(E)=\frac{0.4 Z_{e}+0.1 \alpha+0.7}{E^{0.78}},
$$

where $\sigma_{\text {tot }}$ is the TCS in atomic units $\left(a_{0}^{2}\right), Z_{e}$ is the total number of electrons in the target molecule, $\alpha$ is the static molecular polarizability in atomic units $\left(a_{0}^{3}\right)$, and $E$ is the energy of the incident particles in keV. In Ref. 36, total electron scattering cross sections were successfully predicted for 19 gases ranging from simple diatomic molecules $\left(\mathrm{N}_{2}, \mathrm{O}_{2}\right)$ up to slightly more complex molecules $\left(\mathrm{CH}_{3} \mathrm{~F}, \mathrm{CH}_{3} \mathrm{NH}_{2}\right)$. However, this semi-empirical model has not previously been validated for complex molecules of biological interest (such as ring molecules). In this work, we therefore contrast this model to experimental total cross sections from furfural, THF, and furan and thus check its applicability to larger and more complicated molecules.

\section{RESULTS AND DISCUSSION}

\section{A. Total cross section of furfural}

Although furfural and its parent molecule, furan, have been studied recently by various groups (see Ref. 27 and 
TABLE II. The experimentally measured absolute total scattering cross section, $\sigma_{\text {tot }}(E)$, of furfural (in $\AA^{2}$ ) under the impact of electrons with kinetic energy $E \approx 10-1000 \mathrm{eV}$. The third and the last columns provide the relative statistical and total uncertainties given in per cent, respectively.

\begin{tabular}{|c|c|c|c|}
\hline$E(\mathrm{eV})$ & $\sigma_{\text {tot }}\left(\AA^{2}\right)$ & Statistical uncertainties (\%) & Total uncertainties (\%) \\
\hline 9.17 & 54.29 & 9.29 & 12 \\
\hline 34.0 & 40.14 & 1.38 & 4.37 \\
\hline 71.0 & 43.42 & 4.34 & 7.1 \\
\hline 77.0 & 38.94 & 3.26 & 6.34 \\
\hline 86.0 & 36.34 & 7.16 & 11 \\
\hline 102.0 & 41.31 & 7.64 & 11 \\
\hline 129.0 & 40.82 & 4.78 & 7.72 \\
\hline 169.0 & 38.58 & 0.68 & 3.79 \\
\hline 173.0 & 30.07 & 3.16 & 7.15 \\
\hline 195.0 & 36.26 & 8.12 & 11 \\
\hline 244.0 & 26.53 & 8.29 & 13 \\
\hline 308.0 & 29.80 & 0.41 & 4.43 \\
\hline 308.5 & 25.19 & 6.45 & 11 \\
\hline 354.5 & 23.83 & 8.70 & 14 \\
\hline 433.5 & 21.41 & 5.50 & 13 \\
\hline 496.5 & 20.68 & 6.37 & 14 \\
\hline 524.5 & 20.87 & 4.24 & 11 \\
\hline 603.5 & 13.42 & 5.45 & 17 \\
\hline 704.5 & 13.01 & 1.13 & 13 \\
\hline 820.5 & 13.57 & 8.42 & 19 \\
\hline 929.0 & 12.40 & 7.24 & 19 \\
\hline 967.5 & 12.00 & 8.97 & 22 \\
\hline 970.0 & 12.52 & 2.58 & 15 \\
\hline
\end{tabular}

references therein), the absolute electron impact TCS of furfural has not yet been measured. In Table II, we present the first experimental TCS for electron scattering from furfural in the $\sim 10-1000 \mathrm{eV}$ energy range. In that table, we also provide the associated statistical and total uncertainties on the TCS in terms of being a percentage of that TCS. As described in Sec. II, measurements here have been performed with the endeavor to minimize systematic experimental errors.

Furfural is known to possess a strong permanent dipole moment $(\sim 3.97 \mathrm{D})^{30}$ which suggests that rotational excitations will be an important inelastic scattering channel. Even with the high angular resolution capability of the present double electrostatic analyzer, rotational events, which present DCS values that are very strongly peaked at small scattered electrons angles, will largely not be discriminated against with the present spectrometer. As a consequence, our measured TCS will be lower than those calculated when rotational excitations are taken into account. Thus, to enable a fair comparison between our calculated and measured TCSs, we frame our discussion in terms of comparing our measured TCS against the pure IAM-SCAR+I results, i.e., not including rotational excitations. Figure 4 therefore shows a comparison between our experimental TCS data and our IAM-SCAR+I computational results. In general, we would characterize the level of agreement, over the common energy range, between them as being quite good, with the largest discrepancy being $\sim 29 \%$ at $E=195 \mathrm{eV}$. This difference is clearly outside of the overall experimental uncertainty limits (see Table II). It is also apparent from Fig. 4 that our measured TCS values

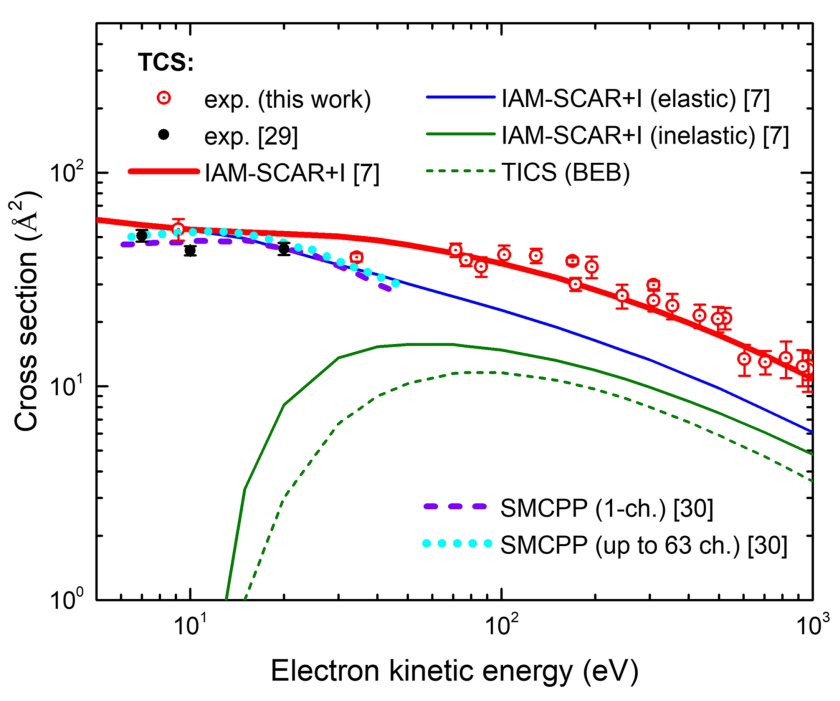

FIG. 4. Present experimentally measured total cross sections of furfural (open symbols) and the earlier results from Lozano et al. ${ }^{29}$ (closed symbols), compared with theoretical calculations employing the IAM-SCAR+I method ${ }^{7}$ without rotations, and the total cross section calculated using the Schwinger multichannel method with pseudopotentials (SMCPP) $)^{30}$ for the elastic and electronically inelastic ICS and the BEB approach ${ }^{56}$ for the ionization cross section (see the text for further details).

scatter more than we would expect for a transmission-beam TCS measurement. ${ }^{55}$ This simply reflects the experimental difficulties in working with such a "sticky" species as furfural and probably is indicative of the observed discrepancy at $E=195 \mathrm{eV}$. Nonetheless, in spite of such experimental difficulties, the TCS data embodied in Fig. 4 constitute an important contribution to the available furfural database. In Fig. 4, we also plot corresponding TCS theoretical results from da Costa et al., ${ }^{30}$ obtained by adding their elastic and electronically inelastic ICSs from the SMCPP approach, at both the 1-channel and 63-channel levels of approximation, to a binary-encounter-Bethe (BEB) total ionization ICS. ${ }^{56}$ Over the somewhat limited energy range $(5-50 \mathrm{eV})$ where such a comparison can be made, agreement between the SMCPP+BEB, IAM-SCAR+I, and present experimental TCS results is quite satisfactory. As expected, the 63-channel SMCPP+BEB result is in better accord with the present measured data than the result from 1-channel SMCPP+BEB computation. It makes good intuitive sense that as more target states are included in the calculation, the calculated values offer a better reproduction of the experimental data. However, note that the SMCPP+BEB 63-channel TCS at $E=34 \mathrm{eV}$ is only about $6 \%$ larger in magnitude than the corresponding 1-channel result. This is a clear indication that the contribution for the excitation of discrete electronic states to the TCS is rather small in furfural, which is entirely consistent with the measurements of Jones et al. ${ }^{23}$

Finally, in Fig. 4 we also plot our IAM-SCAR+I elastic ICS and the ICS for the sum of all the inelastic processes. This enables us to gauge the relative contributions (elastic versus inelastic) that they make to the TCS. For example, at energies below about $50 \mathrm{eV}$, the elastic processes clearly dominate over those for inelastic scattering with this degree of predominance increasing as one goes to lower incident electron energies. 


\section{B. Comparison with other molecules}

To gain further insight into the molecular physicochemical properties governing the energy dependence of the scattering amplitudes, we compare our results for furfural with the data for furan and THF, whose TCSs have been measured by Szmytkowski et al. ${ }^{32}$ and Fuss et al. ${ }^{35}$ respectively. The results of this comparison are shown in Fig. 5. Measurements of the TCS for THF from the work of Fuss et al. ${ }^{35}$ cover the largest energy range as compared to other available experimental studies by Baek et al..$^{34}$ and Zecca et al. ${ }^{33}$ Hence, it is our preferred choice for this comparison.

To counterbalance the historical lack of experimental data, a situation that has improved but remains problematic for many species, various groups have undertaken theoretical developments. A good starting point for quantitative prediction of inelastic scattering is owed to the Born-Bethe theory ${ }^{57}$ and more recent variants of that theory. ${ }^{58}$ The original BornBethe theory is applicable only to fast impinging electrons, with respect to the classical orbiting speeds of the bound electrons. Consequently, it is generally assumed that the BornBethe total scattering probability breaks down below $10 \mathrm{keV},{ }^{16}$ especially for elastic processes, although a number of scaling approaches have revealed its efficiency, for optically allowed excitation ICS, at much lower energies. ${ }^{58}$ Secondary electrons with energies below $10 \mathrm{keV}$ and down to thermal energies eventually carry most of the energy imparted by the incident beam ${ }^{59}$ and so lower energy descriptions are important. Theoretical refinements have thus led to various models, such as the IAM-SCAR+I ${ }^{20}$ the binary-encounter Bethe,${ }^{56}$ the spherical complex optical potential, ${ }^{60}$ or the R-matrix ${ }^{61}$ to name a few, being developed. They all present different methods, of various computational complexity, for predicting the TCS due to electron impact. A computationally cheap method to assess the TCS of complex molecules may be helpful to estimate them, when no experimental or accurate "ab initio" calculated data are available. In Refs. 36 and 54, a simple analytical formula was proposed to calculate the TCS; this formula, Eq. (6), depends only on the number of target electrons and

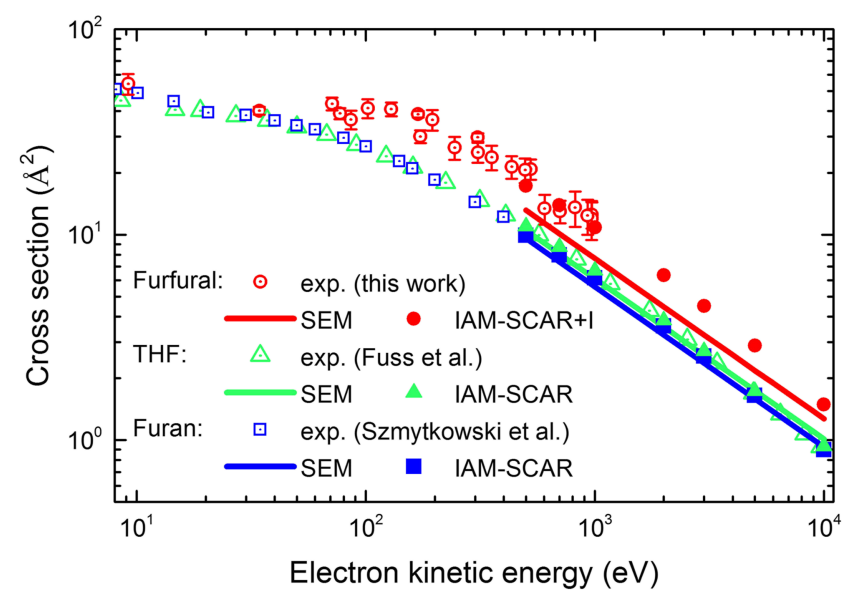

FIG. 5. Total cross sections of furfural, THF, and furan molecules calculated (solid red, green, and blue lines, respectively) using the two-parameter semi-empirical model, Eq. (6), and the IAM-SCAR+I method (filled symbols) for $500 \mathrm{eV}-10 \mathrm{keV}$ electron impact energies. Open symbols represent experimental data from this work and from Refs. 32 and 35. the molecular static polarizability of the species in question. However, this model has never been validated for ring molecular targets possessing higher atomic numbers. In Fig. 5, we contrast results from this semi-empirical model (SEM) to the experimental TCSs of furan, tetrahydrofuran, and furfural. Note that dipole polarizabilities of the considered molecules were obtained from the Computational Chemistry Comparison and Benchmark Database. ${ }^{62}$ According to this database, the dipole polarizabilities, calculated at the MP2 level of theory using the triple-zeta aug-cc-pVTZ basis set, are equal to $66.78 a_{0}^{3}$ (furfural), $48.36 a_{0}^{3}$ (furan), and 51.35 $a_{0}^{3}$ (THF). As shown in Fig. 5, the SEM estimates are of the same order of magnitude as the measurements in each case, offering a simple tool for semi-quantitatively predicting the TCS for energies above about $500 \mathrm{eV}$. Clearly, however, further case studies of other complex molecules are required before we would be able to recommend the SEM approach more generally. Nonetheless, this is worth pursuing as a reliable SEM approach, for energies between 0.5 and $10 \mathrm{keV}$, would be invaluable in radiation interaction simulation studies.

\section{CONCLUSIONS}

Total cross sections for electrons colliding with furfural, in the energy range from $\sim 10$ to $1000 \mathrm{eV}$, have been measured through a double electrostatic hemisphere attenuation experiment. The present experimental results were found to agree, within the uncertainty limits, with its majority of the IAM-SCAR+I theoretical predictions and with results from the Schwinger multichannel method. A comparative study between the present data on furfural, earlier measurements with tetrahydrofuran and furan, and our IAM-SCAR+I results showed a consistent behavior as expected from the additivity rule. A semi-empirical formula for total cross section estimation, depending on the number of target electrons and the molecular polarizability, was also applied to these ring molecules, exhibiting a level of accord that was a little less conclusive although still promising from a semi-quantitative perspective.

Finally, in terms of accurately modeling electron transport through furfural, we note that further work that extends our knowledge of the interaction cross sections from the different scattering processes to lower energies, in particular for rotational and vibrational excitations, is highly desirable.

\section{ACKNOWLEDGMENTS}

We acknowledge the financial support received from the European Union Seventh Framework Programme (PEOPLE2013-ITN-ARGENT Marie Curie project), under Grant Agreement No. 608163, and from the Spanish Ministerio de Economia y Competitividad (Project No. FIS2016-80440). M.J.B. also acknowledges the Australian Research Council for some financial support. G.G. acknowledges M. J. HubinFranskin and J. Delwiche for providing the double spectrometer apparatus that constituted the basis of the present experimental study. 
${ }^{1}$ K. J. Zeitsch, The Chemistry and Technology of Furfural and its Many By-Products, 1st ed. (Elsevier Science, 2000).

${ }^{2}$ A. S. Mamman, J.-M. Lee, Y.-C. Kim, I. T. Hwang, N.-J. Park, Y. K. Hwang, J.-S. Chang, and J.-S. Hwang, Biofuels, Bioprod. Biorefin. 2, 438 (2008).

${ }^{3}$ H. Kobayashi and A. Fukuoka, Green Chem. 15, 1740 (2013).

${ }^{4}$ A. W. Khan, J.-P. Labrie, and J. McKeown, Biotechnol. Bioeng. 28, 1449 (1986).

${ }^{5}$ J. Amorim, C. Oliveira, J. A. Souza-Corrêa, and M. A. Ridenti, Plasma Processes Polym. 10, 670 (2013).

${ }^{6}$ M. A. Ridenti, J. A. Filho, M. J. Brunger, R. F. da Costa, M. T. do N. Varella, M. H. F. Bettega, and M. A. P. Lima, Eur. Phys. J. D 70, 161 (2016).

${ }^{7}$ D. B. Jones, R. F. da Costa, M. T. do N. Varella, M. H. F. Bettega, M. A. P. Lima, F. Blanco, G. García, and M. J. Brunger, J. Chem. Phys. 144, 144303 (2016).

${ }^{8}$ J. Tennyson et al., Plasma Sources Sci. Technol. 26, 055014 (2017).

${ }^{9}$ D. B. Jones, R. F. C. Neves, M. C. A. Lopes, R. F. da Costa, M. T. do N. Varella, M. H. F. Bettega, M. A. P. Lima, G. García, F. Blanco, and M. J. Brunger, J. Chem. Phys. 143, 224304 (2015).

${ }^{10}$ L. Chiari, E. Anderson, W. Tattersall, J. R. Machacek, P. Palihawadana, C. Makochekanwa, J. P. Sullivan, G. García, F. Blanco, R. P. McEachran, M. J. Brunger, and S. J. Buckman, J. Chem. Phys. 138, 074301 (2013).

${ }^{11}$ S. M. Pimblott and J. A. LaVerne, Radiat. Phys. Chem. 76, 1244 (2007).

${ }^{12}$ E. Alizadeh, T. M. Orlando, and L. Sanche, Annu. Rev. Phys. Chem. 66, 379 (2015).

${ }^{13}$ L. Sanche, Mass Spectrom. Rev. 21, 349 (2002).

${ }^{14}$ K. Bartschat and M. J. Kushner, Proc. Natl. Acad. Sci. U. S. A. 113, 7026 (2016).

${ }^{15}$ A. G. Sanz, M. C. Fuss, A. Muñoz, F. Blanco, P. Limão-Vieira, M. J. Brunger, S. J. Buckman, and G. García, Int. J. Radiat. Biol. 88, 71 (2012).

${ }^{16}$ F. Blanco, A. Muñoz, D. Almeida, F. F. da Silva, P. Limão-Vieira, M. C. Fuss, A. G. Sanz, and G. García, Eur. Phys. J. D 67, 199 (2013).

${ }^{17}$ M. J. Brunger and P. J. O. Teubner, Phys. Rev. A 41, 1413 (1990).

${ }^{18}$ W. M. Ariyasinghe and D. Powers, Phys. Rev. A 66, 052716 (2002).

${ }^{19}$ M. C. Fuss, A. G. Sanz, F. Blanco, J. C. Oller, P. Limão-Vieira, M. J. Brunger, and G. García, Phys. Rev. A 88, 042702 (2013).

${ }^{20}$ F. Blanco, L. Ellis-Gibbings, and G. García, Chem. Phys. Lett. 645, 71 (2016).

${ }^{21}$ W. Garrett, Phys. Rev. A 11, 1297 (1975).

${ }^{22}$ J. Tennyson, Phys. Rep. 491, 29 (2010).

${ }^{23}$ D. B. Jones, R. F. C. Neves, M. C. A. Lopes, R. F. da Costa, M. T. do N. Varella, M. H. F. Bettega, M. A. P. Lima, G. García, P. Limão-Vieira, and M. J. Brunger, J. Chem. Phys. 144, 124309 (2016).

${ }^{24}$ D. B. Jones, E. Ali, K. L. Nixon, P. Limão-Vieira, M.-J. Hubin-Franskin, J. Delwiche, C. G. Ning, J. Colgan, A. J. Murray, D. H. Madison, and M. J. Brunger, J. Chem. Phys. 143, 184310 (2015).

${ }^{25}$ F. Ferreira da Silva, E. Lange, P. Limão-Vieira, N. C. Jones, S. V. Hoffmann, M.-J. Hubin-Franskin, J. Delwiche, M. J. Brunger, R. F. C. Neves, M. C. A. Lopes, E. M. de Oliveira, R. F. da Costa, M. T. do N. Varella, M. H. F. Bettega, F. Blanco, G. García, M. A. P. Lima, and D. B. Jones, J. Chem. Phys. 143, 144308 (2015).

${ }^{26}$ S. Marjanović, A. Banković, R. D. White, S. J. Buckman, G. García, G. Malović, S. Dujho, and Z. Lj Petrović, Plasma Sources Sci. Technol. 24, 025016 (2015).

${ }^{27}$ M. J. Brunger, Int. Rev. Phys. Chem. 36, 333 (2017).

${ }^{28} \mathrm{~S}$. Trajmar and D. Cartwright, "Excitation of molecules by electron impact," in Electron-Molecule Interactions and their Applications, edited by L. Christophorou (Elsevier, 1984), pp. 155-249.

${ }^{29}$ A. I. Lozano, K. Krupa, F. Ferreira da Silva, P. Limão-Vieira, F. Blanco, A. Muñoz, D. B. Jones, M. J. Brunger, and G. García, "Low energy electron transport in furfural," Eur. Phys. J. D. (in press).
${ }^{30}$ R. F. da Costa, M. T. do N. Varella, M. H. F. Bettega, R. F. C. Neves, M. C. A. Lopes, F. Blanco, G. García, D. B. Jones, M. J. Brunger, and M. A. P. Lima, J. Chem. Phys. 144, 124310 (2016).

${ }^{31}$ P. Mozejko, E. Ptasińska-Denga, A. Domaracka, and C. Szmytkowski, Phys. Rev. A 74, 012708 (2006).

${ }^{32}$ C. Szmytkowski, P. Mozzejko, E. Ptasińska-Denga, and A. Sabisz, Phys. Rev. A 82, 032701 (2010).

${ }^{33}$ A. Zecca, C. Perazzolli, and M. J. Brunger, J. Phys. B: At., Mol. Opt. Phys. 38, 2079 (2005).

${ }^{34}$ W. Y. Baek, M. Bug, H. Rabus, E. Gargioni, and B. Grosswendt, Phys. Rev. A 86, 032702 (2012).

${ }^{35}$ M. Fuss, A. Muñoz, J. Oller, F. Blanco, D. Almeida, P. Limão-Vieira, T. P. D. Do, M. J. Brunger, and G. García, Phys. Rev. A 80, 052709 (2009).

${ }^{36}$ G. García and F. Manero, Chem. Phys. Lett. 280, 419 (1997).

${ }^{37}$ C. Hannay, J. Heinesch, U. Kleyens, and M.-J. Hubin-Franskin, Meas. Sci. Technol. 6, 1140 (1995).

${ }^{38}$ D. Duflot, J.-P. Flament, A. Giuliani, J. Heinesch, M. Grogna, and M.-J. Hubin-Franskin, Phys. Rev. A 75, 052719 (2007).

${ }^{39}$ D. Duflot, J.-P. Flament, A. Giuliani, J. Heinesch, and M.-J. Hubin-Franskin, Chem. Phys. 310, 67 (2005).

${ }^{40}$ J. H. Moore, C. C. Davis, M. A. Coplan, and S. C. Greer, Building Scientific Apparatus (Cambridge University Press, 2009).

${ }^{41}$ M.-J. Hubin-Franskin, H. Aouni, D. Duflot, F. Motte-Tollet, C. Hannay, L. F. Ferreira, and G. Tourillon, J. Chem. Phys. 106, 35 (1997).

${ }^{42}$ E. Gargioni and B. Grosswendt, Rev. Mod. Phys. 80, 451 (2008).

${ }^{43}$ F. Blanco and G. García, Phys. Rev. A 67, 022701 (2003).

${ }^{44}$ F. Blanco and G. García, Phys. Lett. A 330, 230 (2004).

${ }^{45}$ F. Blanco, J. Rosada, A. Illana, and G. García, Phys. Lett. A 374, 4420 (2010).

${ }^{46}$ M. C. Fuss, L. Ellis-Gibbings, D. B. Jones, M. J. Brunger, F. Blanco, A. Muñoz, P. Limão-Vieira, and G. García, J. Appl. Phys. 117, 214701 (2015).

${ }^{47}$ R. D. Cowan, The Theory of Atomic Structure and Spectra, Los Alamos Series in Basic and Applied Sciences (University of California Press, 1981).

${ }^{48}$ M. E. Riley and D. G. Truhlar, J. Chem. Phys. 63, 2182 (1975).

${ }^{49}$ X. Zhang, J. Sun, and Y. Liu, J. Phys. B: At., Mol. Opt. Phys. 25, 1893 (1992).

${ }^{50}$ G. Staszewska, D. W. Schwenke, D. Thirumalai, and D. G. Truhlar, Phys. Rev. A 28, 2740 (1983).

${ }^{51}$ F. Blanco and G. García, Phys. Lett. A 295, 178 (2002).

${ }^{52}$ O. Zatsarinny, K. Bartschat, G. García, F. Blanco, L. R. Hargreaves, D. B. Jones, R. Murrie, J. R. Brunton, M. J. Brunger, M. Hoshino, and S. J. Buckman, Phys. Rev. A 83, 042702 (2011).

${ }^{53}$ F. Blanco, A. M. Roldán, K. Krupa, R. P. McEachran, R. D. White, S. Marjanović, Z. Lj. Petrović, M. J. Brunger, J. R. Machacek, S. J. Buckman, J. P. Sullivan, L. Chiari, P. Limão-Vieira, and G. García, J. Phys. B: At., Mol. Opt. Phys. 49, 145001 (2016).

${ }^{54}$ G. García and F. Blanco, Phys. Lett. A 279, 61 (2001).

${ }^{55}$ M. J. Brunger and S. J. Buckman, Phys. Rep. 357, 215 (2002).

${ }^{56}$ W. Hwang, Y.-K. Kim, and M. E. Rudd, J. Chem. Phys. 104, 2956 (1996).

${ }^{57}$ M. Inokuti, Rev. Mod. Phys. 43, 297 (1971).

${ }^{58}$ H. Tanaka, M. J. Brunger, L. Campbell, H. Kato, M. Hoshino, and A. R. P. Rau, Rev. Mod. Phys. 88, 025004 (2016).

${ }^{59}$ S. M. Pimblott and A. Mozumder, J. Phys. Chem. 95, 7291 (1991).

${ }^{60}$ A. Jain, J. Chem. Phys. 86, 1289 (1987).

${ }^{61}$ L. A. Morgan, J. Tennyson, and C. J. Gillan, Comput. Phys. Commun. 114, 120 (1998).

${ }^{62}$ Computational Chemistry Comparison and Benchmark DataBase, NIST Standard Reference Database No. 101, Release 18, edited by R. D. Johnson III (NIST, 2016), http://cccbdb.nist.gov/. 